\title{
Modelling Hydromechanical Dilation Geomaterial-Cavitation and Localization
}

\author{
Y. Sieffert, O. Buzzi, F. Collin and R. Chambon
}

\begin{abstract}
This paper presents an extension of the local second gradient model to multiphasic materials (solid particles, air, water) and including the cavitation phenomenon. This new development was made in order to model the response of saturated dilatant materials under deviatoric stress and undrained conditions and possibly, in future, the behaviour of unsaturated soils.
\end{abstract}

\section{Introduction}

A characteristic of geomaterials is to develop inelastic volume change. Clays, sands, rocks and concrete are dilatant materials, i.e. the porosity increases in the plastic regime.

In the case of saturated state, the pores are saturated with fluid. With a material permeability dependence, the pore volume increases more rapidly than the fluid can flow inside. Then the fluid is in tension which leads to a decrease of the pore pressure until negative pore pressure could be achieved.

This well-known problem in numerical modelling leads to a dilatant hardening behaviour because the decrease of pore water pressure is coupled with an increase of effective stresses (compression). This is particularly problematic with constitutive

Y. Sieffert $(\triangle) \cdot R$. Chambon

Laboratoire 3S-R, Grenoble-INP, CNRS, Grenoble Université Joseph-Fourier,

B.P. 53X, 38041 Grenoble Cedex, France

e-mail: yannick.sieffert@3sr-grenoble.fr

O. Buzzi

Priority Research Centre for Geotechnical and Materials Modelling,

The University of Newcastle, Callaghan, NSW, Australia

F. Collin

Department of ArGEnCo Institut de Mécanique et Génie Civil, Université de Liège,

1 Chemin Des Chevreuils, 4000 Liège, Belgium 
equations modelling the degradation of the strength of materials as pressure of pore water may have an influence on shear band formation (Loret and Prevost 1991).

\section{Presentation of the Model}

Before starting, it is important to specify the main limitations of this work. The first restriction of this study is that we deal only with quasi-static problems in unsaturated conditions, under Richard's assumptions (vapour water pressure is constant). Furthermore for the sake of simplicity, isothermal condition, incompressible solid grains, incompressible vapour water, no flow for gas (no entry of air) are assumed. However phase changes between fluid and vapour water are considered. In all the computation large strains effects are taken into account.

As in Collin et al. (2006), the pore fluid and water vapour are assumed no influence at the microstructure level, micro kinematic gradient is not generated by pore pressure and vapour water variations.

The unknowns of the second gradient mechanical and the flow problems are respectively the (macro) displacement $u_{i}$, the micro kinematic $v_{i j}$ and the pore water pressure $p^{w}$ (possibly negative in unsaturated case).

In order to get second gradient models, we add the assumption that the micro kinematic gradient $v_{i j}$ is equal to the macro displacement gradient $F_{i j}$. This implies similar relations for virtual quantities.

$$
v_{i j}^{*}=\frac{\partial u_{i}^{*}}{\partial_{x j}}=F_{i j}^{*}
$$

In the framework of Schrefler's stress, the effective stress is:

$$
\sigma_{i j}^{t}=\sigma_{i j}^{\prime t}+S_{r}^{w, t} p^{w, t} \delta_{i j}+\left(1-S_{r}^{w, t}\right) p^{v} \delta_{i j}
$$

$\sigma_{i j}^{t}$ is the total stress, $\sigma_{i j}^{\prime t}$ is the effective stress, $p^{w, t}$ is the fluid pressure, $p^{v}$ is the vapour pressure, $S_{r}^{w, t}$ is the water relative saturation and $\delta_{i j}$ is Kronecker's delta.

$$
S_{r}^{w, t}+S_{r}^{v, t}=1
$$

$S_{r}^{v, t}$ is the vapour relative saturation.

The mass density of the mixture is:

$$
\varrho^{m i x, t}=\varrho^{s}\left(1-\phi^{t}\right)+S_{r}^{w, t} \varrho^{w, t} \phi^{t}+\left(1-S_{r}^{w, t}\right) \varrho^{v} \phi^{t}
$$

$\varrho^{S}$ is the solid grain density (assumed to be incompressible, i.e. $\varrho^{S}=$ cte), $\varrho^{w, t}$ is the fluid density, $\phi^{t}$ is the porosity defined as $\phi^{t}=\Omega^{p, t} / \Omega^{t}$ where $\Omega^{t}$ is the current volume of a given mass of skeleton and $\Omega^{p, t}$ the corresponding porous volume.

In weak form (virtual work principle), the momentum balance for the mixture can thus be written as: 


$$
\int_{\Omega^{t}}\left(\sigma_{i j}^{t} \frac{\partial u_{i}^{*}}{\partial x_{j}^{t}}+\sum_{i j k}^{t} \frac{\partial^{2} u_{i}^{*}}{\partial x_{j}^{t} \partial x_{k}^{t}}\right) d \Omega^{t}=\int_{\Omega^{t}} \varrho^{m i x, t} g_{i} u_{i}^{*} d \Omega^{t}+\int_{\Gamma_{\Omega}^{t}}\left(\overline{t_{\iota}} u_{i}^{*}+\overline{T_{\imath}} D u_{i}^{*}\right) d \Gamma^{t}
$$

where $u_{i}^{*}$ is any kinematically admissible virtual displacement field, $\sigma_{i j}^{t}$ is the Cauchy stress (total stress), $\sum_{i j k}^{t}$ is the double stress dual of the virtual second micro kinematic gradient, $x_{i}$ are the current coordinates, $g_{i}$ is the gravity acceleration, $\bar{t}_{i}$ is the external (classical) forces per unit area and $\bar{T}_{i}$ an additional external (double) force per unit area, both applied on a part $\Gamma_{\Omega}^{t}$ of the boundary of $\Omega^{t} \cdot D$ denotes the normal derivative of any quantity $q$.

In order to use $C^{0}$ functions for the displacement field (i.e. only first derivatives of the displacement), the following equation with $\lambda_{i j}$ Lagrange multipliers is used.

$$
\begin{gathered}
\int_{\Omega^{t}}\left(\sigma_{i j}^{t} \frac{\partial u_{i}^{*}}{\partial x_{j}^{t}}+\sum_{i j k}^{t} \frac{\partial v_{i j}^{*}}{\partial x_{k}^{t}}\right) d \Omega^{t}-\int_{\Omega^{t}} \lambda_{i j}^{t}\left(\frac{\partial u_{i}^{*}}{\partial x_{j}^{t}}-v_{i j}^{*}\right) d \Omega^{t} \\
=\int_{\Omega^{t}} \varrho^{m i x, t} g_{i} u_{i}^{*} d \Omega^{t}+\int_{\Gamma_{\Omega}^{t}}\left(\overline{t_{i}} u_{i}^{*}+\bar{T} D u_{i}^{*}\right) d \Gamma^{t}, \\
\int_{\Omega^{t}} \lambda_{i j}^{*}\left(\frac{\partial u_{i}^{t}}{\partial x_{j}^{t}}-v_{i j}^{t}\right) d \Omega^{t}=0
\end{gathered}
$$

In volume $\Omega^{t}$, the liquid fluid mass is equal to $M_{\Omega^{t}}^{w, t}=S_{r}^{w, t} \varrho^{w, t} \phi^{t} \Omega^{t}$ and the vapour fluid mass is equal to $M_{\Omega^{t}}^{w, t}=S_{r}^{v, t} \varrho^{v} \phi^{t} \Omega^{t}$.

In weak form, the mass balance equation for the fluid and water vapour can thus be written as:

$$
\begin{aligned}
& \int_{\Omega^{t}}\left(\left(\dot{M}^{w, t}+\dot{M}^{v, t}\right) p^{w^{*}}-\mathrm{m}_{i}^{w, t} \frac{\partial p^{*}}{\partial x_{i}^{t}}\right) d \Omega^{t} \\
& =\int_{\Omega^{t}} Q^{w, t} p^{w^{*}} d \Omega^{t}-\int_{\Gamma_{q}^{t}} \bar{q}^{w, t} p^{w^{*}} d \Gamma^{t}, \\
& \dot{M}^{w, t}=\varrho^{w, t}\left(\frac{\dot{p}^{w, t}}{k^{w}} S_{r}^{w, t} \phi^{t}+\dot{S}_{r}^{w, t} \phi^{t}-S_{r}^{w, t} \frac{\dot{\Omega}^{t}}{\Omega^{t}}\right), \\
& \dot{M}^{v, t}=\varrho^{v}\left(-\left(1-S_{r}^{w, t}\right) \frac{\dot{\Omega}^{t}}{\Omega^{t}}-\dot{S}_{r}^{w, t} \phi^{t}\right), \\
& m_{i}^{w, t}=-\varrho^{w, t} k \frac{k_{r}^{w, \tau_{1}}}{\mu^{W}}\left(\frac{\partial p^{w, t}}{\partial x_{i}^{t}}+\varrho^{w, t} g_{i}\right)
\end{aligned}
$$

With $\dot{M}^{w, t}$ is the time derivative of the fluid phase, $m_{i}^{w, t}$ is the mass flow of water, $k^{w}$ is the fluid bulk modulus, $k$ is the intrinsic permeability, $k_{r}^{w, t}$ is the water 
relative permeability, $\mu^{w}$ is the fluid viscosity, $Q^{w, t}$ is a sink term and $\Gamma_{q}^{t}$ is the part of the boundary where the input fluid mass per unit area $\bar{q}^{w, t}$ is prescribed.

\subsection{Mechanical Constitutive Law}

In order to reproduce the progressive decrease of the material strength, the mechanic constitutive law used in this study is an elastoplastic strain-softening Druker-Prager model with yield criterion given by the follow equation (Barnichon 1998):

$$
f=I I_{\widehat{\sigma}}-m \mathrm{I}_{\sigma}+k=0
$$

where $m$ and $k$ is defined by: $m=\frac{2 \sin \phi_{c}}{\sqrt{3}\left(3-\sin \phi_{c}\right)}, k=\frac{6 c \cos \phi_{c}}{\sqrt{3}\left(3-\sin \phi_{c}\right)} . \phi_{c}$ is the friction angle, $c$ is the cohesion, $I_{\sigma}=\sigma_{i i}$ is the first invariant and $I I_{\widehat{\sigma}}=\sqrt{\frac{1}{2} \widehat{\sigma}_{i j} \widehat{\sigma}_{i j}}$ is the second deviatoric invariant.

A general non-associated plasticity framework is considered to define the rate of plastic flow as perpendicular to the plastic potential $\mathrm{g}: \dot{\varepsilon}_{i j}^{p}=\dot{\lambda} \frac{\partial g}{\partial \sigma_{i j}}$ and $g=I I_{\widehat{\sigma}}-m^{\prime} \mathrm{I}_{\sigma}=C_{1}$ with $m^{\prime}=\frac{2 \sin \psi}{\sqrt{3}(3-\sin \psi)}$ with, $\psi$ is the dilatancy angle and $c_{1}$ is a constant. The softening process during plastic flow is introduced via an hyperbolic variation of the cohesion between initial $c_{0}$ and final $c_{f}$ values as a function of the Von Mises equivalent plastic strain $\varepsilon_{e q}^{p}: c=c_{0}+\frac{\left(c_{f}-c_{0}\right) \varepsilon_{e q}^{p}}{\beta_{c}+\varepsilon_{e q}^{p}}$.

\subsection{Model Parameters}

The Drucker-Prager model presents the advantage to use simple formulation and does not require enough parameters. All parameter values are presented in Tables 1 and 2.

Table 1 Mechanic parameters

\begin{tabular}{l|l}
\hline Geomechanical characteristics & \\
\hline Young's elastic modulus $(\mathrm{MPa})$ & 300 \\
\hline Poisson's ratio $(-)$ & 0.125 \\
\hline Initial cohesion $(\mathrm{kPa}) c_{0}$ & 300 \\
\hline Final cohesion $(\mathrm{kPa}) c_{f}$ & 100 \\
\hline Softening parameter $(-) \beta_{c}$ & 0.01 \\
\hline Friction angle $\left(^{\circ}\right) \phi_{c}$ & 18 \\
\hline Dilation angle $\left(^{\circ}\right) \psi$ & 10 \\
\hline Solid specific mass $\left(\mathrm{kg} / \mathrm{m}^{3}\right) \varrho^{s}$ & 2,026 \\
\hline Second gradient parameter $(\mathrm{N}) D$ & 150 \\
\hline
\end{tabular}


Table 2 Hydraulic and air parameters

\begin{tabular}{l|l}
\hline Hydraulic characteristics & \\
\hline Initial porosity $(-) \phi$ & 0.39 \\
\hline Intrinsic permeability $(\mathrm{m} / \mathrm{s}) k$ & $10^{-7}$ \\
\hline Initial relative water permeability $(-)$ & 1 \\
\hline Water specific mass $\left(\mathrm{kg} / \mathrm{m}^{3}\right) \varrho^{w}$ & 1,000 \\
\hline Water dynamic viscosity $(\mathrm{Pa} \cdot \mathrm{s}) \mu^{w}$ & $10^{-3}$ \\
\hline Water compressibility coefficient $\left(\mathrm{MPa}^{-1}\right) 1 / k^{w}$ & $5.10^{-4}$ \\
\hline Air characteristics & -100 \\
\hline Gas pressure $(\mathrm{kPa}) p^{v}$ &
\end{tabular}

\subsection{Cavitation Model}

In the model, before cavitation, the stress state governs the pore pressure and the specimen is saturated. After cavitation, a phase change takes place and the pore pressure is related to the relative degree of saturation as per the cavitation equation below: $p^{w, t}=p_{c}-C_{2}+C_{2} \cdot \exp ^{-\left(\frac{1-\left(s_{r}^{w, t} / 100\right)}{0.02}\right)}$ for $p^{w, t}<p_{c}$ with $C_{2}=40 \mathrm{kPa}$ in this paper.

\section{Results}

Visualization of the localization shear bands is performed by observing the loading index of a Gauss point for a given time step. When a Gauss point undergoes a plastic loading, a small square is plotted. No marker appears if the element undergoes elastic loading or unloading (Fig. 1).

The numerical results are displayed in the form of load-displacement curve and pore pressure inside the shear band curve in Fig. 2. First, the biaxial test leads to a homogenous solution, i.e. the plasticity behavior is identical in the entire sample (Fig. 1a). As all elements of the model enter into plasticity, global dilation takes place and pore pressure starts dropping steadily. The reduction in pore pressure turns into an increase of effective stress. The homogeneous solution is stable until the pore pressure achieves the cavitation pressure. Then, the water starts to change in vapour phase (Figs. 1b and 2). The effective stresses can decrease freely and localized bands are obtained. In accord with the experimental results given by Mokni and Desrues (1998), when the plasticity is first obtained with an homogeneous behavior, the cavitation triggers strain localization and then cavitation occurs before localization. 

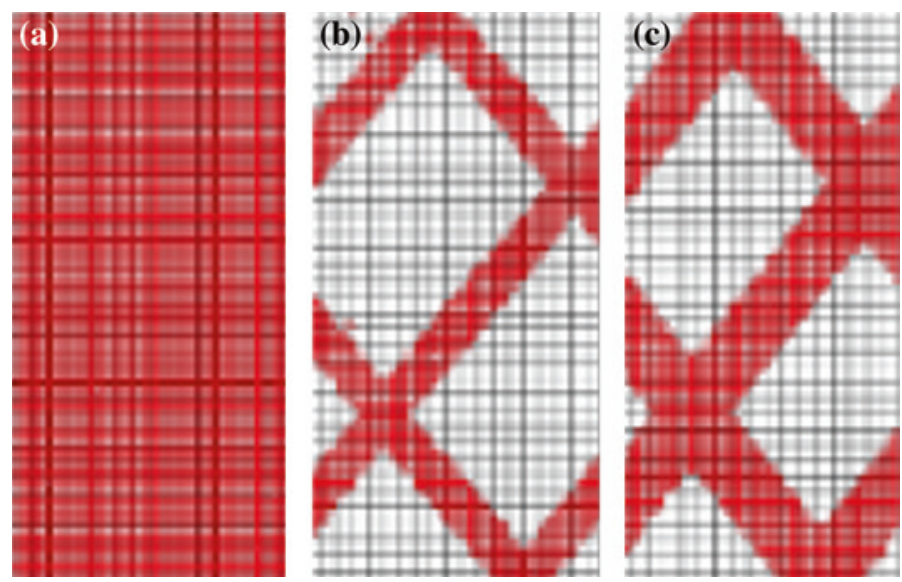

Fig. 1 Visualization of the shear bands using the loading index at the gauss point a axial strain $=0.14 \%, \mathbf{b}$ axial strain $=1.8 \%$, and $\mathbf{c}$ axial strain $=2.5 \%$

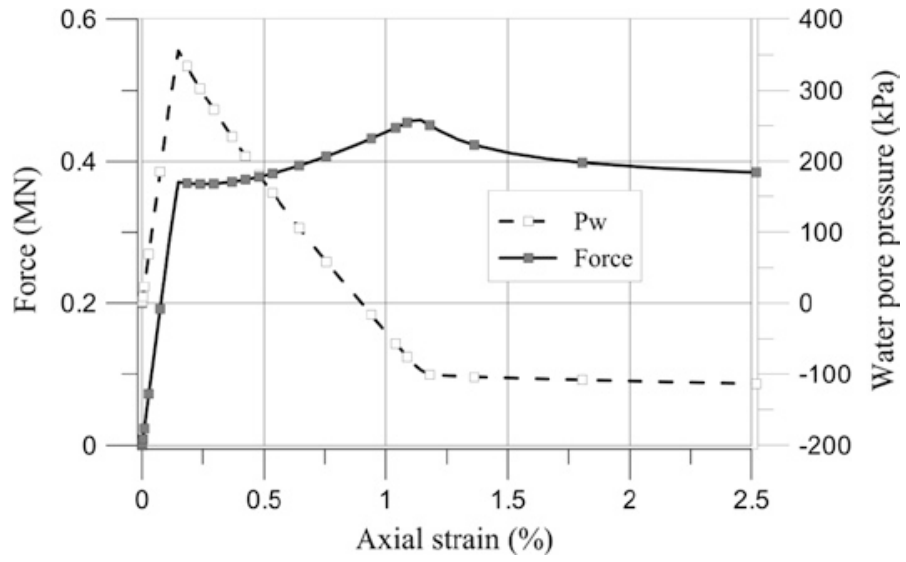

Fig. 2 Global curves of the loading force and water pressure versus the specimen shortening

\section{Conclusion}

This paper presents numerical investigations of strain localization for a dilatant material. To ensure objectivity of the formulation, i.e. no mesh dependency, a second gradient hydromechanical model is used. This paper gives a numerical implementation of the cavitation phenomena to resolve the challenge of the unrealistic negative pore pressure obtained typically with a dilatant porous material which could lead at a global hardening response. Based on the numerical simulation, the cavitation is capable of restoring the shear bands formation. 


\section{References}

Barnichon J (1998) Finite element modelling in structural and petroleum geology. Ph.D. thesis, University of Liège, Belgium

Collin F, Chambon R, Charlier R (2006) A finite element method for poro mechanical modelling of geotechnical problems using local second gradient models. Int J Numer Methods Eng 65:1749-1772

Loret B, Prevost JH (1991) Dynamics strain localisation in fluid-saturated porous media. J Eng Mech 11:907-922

Mokni M, Desrues J (1998) Strain localization measurements in undrained plane-strain biaxial tests on Hoston RF sand. Mech Cohesive-Frictional Mater Struct 4:419-441 